\title{
SAR TOMOGRAPHY FOR SPATIO-TEMPORAL INVERSION OF COHERENT SCATTERERS IN VILLAGES OF ALPINE REGIONS
}

\author{
Muhammad A. Siddique ${ }^{1}$, Tazio Strozzi $i^{2}$, Irena Hajnsek ${ }^{1,3}$, Othmar Frey ${ }^{1,2}$ \\ ${ }^{1}$ Earth Observation and Remote Sensing, ETH Zurich, Switzerland \\ ${ }^{2}$ Gamma Remote Sensing AG, Gümligen, Switzerland \\ ${ }^{3}$ Microwaves and Radar Institute, German Aerospace Center - DLR, Oberpfaffenhofen, Germany
}

\begin{abstract}
Differential synthetic aperture radar (SAR) tomography allows separation of multiple coherent scatterers interfering in the same range-azimuth resolution cell as well as the estimation of the deformation parameters of each scatterer. In this way, the spatio-temporal tomographic inversion serves as a means to resolve the layover and simultaneously improve deformation sampling. Compared to metropolitan regions with several man-made structures, the prevalence of coherent scatterers in the villages of alpine regions is generally low, while at the same time layovers are widespread due to the ruggedness of the terrain. Moreover, the drastic height variations in the imaged scene necessitate height-dependent compensation of the atmospheric phase delay variations within the tomographic inversion. This paper addresses these concerns while performing experiments on an interferometric stack comprising 33 Cosmo-SkyMed strimap images acquired in the summers between 2008-13 over Matter Valley in the Swiss Alps. The results show improved deformation sampling along the layover-affected mountainside.
\end{abstract}

Index Terms - SAR tomography, persistent scatterer interferometry, multi-baseline interferometry, Cosmo-SkyMed

\section{INTRODUCTION}

Persistent scatterer interferometry (PSI) $[1,2]$ is a remote sensing technique for the estimation of deformation time series using interferometric SAR data stacks. A restriction associated with PSI is that the spatio-temporal inversion of the observed interferometric phases to retrieve deformation estimates is limited to the so-called persistent scatterers (PS), i.e. single dominant and temporally phase coherent scatterers. In other words, the range-azimuth resolution cells containing multiple coherent scatterers are typically rejected in the PSI

This research project has been partly funded by the Swiss Space Office, State Secretariat for Education and Research of the Swiss Confederation (SER/SSO), via the MdP2012 initiative. The DEM used in this work is Oswisstopo (JD100042). The data stack used in this work is a COSMOSkyMed Product - ASI - Agenzia Spaziale Italiana - (2013). All Rights Reserved. processing, which represent a missed opportunity to get more information about the underlying anthropogenic or natural deformation process. Compared to metropolitan regions with several man-made structures, the prevalence of coherent scatterers in alpine regions is already low, while at the same time layovers are generally more widespread due to the ruggedness of the topography. Settlements and other infrastructure in the valleys are often partly and sometimes completely in layover cast by the adjoining mountain(s). Moreover, mass movements of interest such as landslides and rockfalls often take place in mountainous regions. Timely deformation measurements on slopes close to the villages can potentially assist in preventing untoward incidents. These concerns motivate this investigation on the potential of differential SAR tomography $[3,4,5]$ as a means to resolve the layovers and allow spatio-temporal inversion of individually coherent scatterers interfering in the same resolution cell. The prospects of SAR tomography in alpine regions come across several challenges. Among them, a particularly complex issue is the phase calibration of the interferometric stack as a prerequisite for tomographic inversion. The refractivity of the troposphere changes spatially over the scene as well as from one pass to the next, incurring variable phase delays which in general do not cancel out in interferogram formation, leaving behind a phase footprint, i.e the atmospheric phase. It acts as a disturbance in focusing the scatterers in 3-D [6, 7] and needs to be corrected. In mountainous regions, the local atmospheric conditions and the propagation paths through the troposphere may strongly vary spatially due to the extremely rugged topography which may change by as much as a few kilometers between the valley floor and the mountain top. Therefore, the atmospheric correction in such areas is more involved.

\section{METHODS}

The methodology proposed in this work entails a prior PSI analysis, which we perform using the Interferometric Point Target Analysis (IPTA) [2] toolbox. A set of PS is iteratively identified using least squares regression [8]. The atmospheric phases for these PS are estimated within the PSI processing 
by isolating these components from other phase contributions (e.g. residual topography and deformation) with appropriate spatio-temporal filtering and unwrapping at each iteration. In the case of alpine regions, the drastic variations in topography incur a height-dependent phase delay variation owing to vertical stratification of the atmosphere. We address this concern by fitting a linear model for the dependence of unwrapped atmospheric phases on topography during the iterative PSI processing $[9,10]$.

\subsection{3-D Phase calibration}

Depending on the acquisition geometry, the possibly large height differences among the scatterers in layover (e.g. between the scatterers in the valley with those on the rocky mountainsides) implies that the spatial location of the individual scatterers in map coordinates (after geocoding) can also be very different. In this case, the atmospheric correction needed for one scatterer may be very different from the other, although they may be superposed in the same rangeazimuth pixel. Hence, a single correction for a range-azimuth pixel does not suffice; instead, the atmospheric phase correction has to be estimated and applied within the tomographic focusing at each 3-D point of interest along the elevation axis.

Considering the atmospheric phases estimated at the PS locations (in map geometry) as samples of the 3-D distribution of the atmospheric phase delay variations over the entire scene, we use regression-kriging (a.k.a. universal kriging) to predict the atmospheric phase at a given location. The following multiple regression model is assumed:

$$
\psi_{m}(\boldsymbol{x})=\mathbf{x} \boldsymbol{\beta}_{m}+\varepsilon_{m}(\boldsymbol{x})
$$

where $\psi_{m}$ represents the unwrapped atmospheric phase for the $m^{\text {th }}$ interferometric layer, and $\mathbf{x}=\left[\begin{array}{cccc}1 & x_{e} & x_{n} & h\end{array}\right]$. $\boldsymbol{x} \triangleq\left(x_{e}, x_{n}, h\right)=\mathbb{T}\{r, a, s\}$ represents a general 3-D location in map geometry in terms of easting, $x_{e}$, northing, $x_{n}$ and height, $h . \mathbb{T}\{\cdot\}$ is the geocoding transformation applied on a range-azimuth-elevation tuple $(r, a, s) . \varepsilon_{m}$ denotes the residue of the fit. The regression coefficients are contained in the vector $\boldsymbol{\beta}_{\boldsymbol{m}}$. They are estimated with generalized least squares [11]:

$$
\hat{\boldsymbol{\beta}}_{\boldsymbol{m}}=\left(\mathbf{X}^{T} \mathbf{V}_{m}^{-1} \mathbf{X}\right)^{-1} \mathbf{X}^{T} \mathbf{V}_{m}^{-1} \Psi_{m}
$$

where $\mathbf{X}$ is the design matrix and $\boldsymbol{\Psi}_{m}$ is the vector of the atmospheric phases at PS locations:

$$
\mathbf{X}=\left[\begin{array}{c}
\mathbf{x}_{1}^{T} \\
\mathbf{x}_{2}^{T} \\
\vdots \\
\mathbf{x}_{N_{\mathrm{ps}}}^{T}
\end{array}\right], \quad \boldsymbol{\Psi}_{m}=\left[\begin{array}{c}
\psi_{0}\left(\boldsymbol{x}_{1}\right) \\
\psi_{1}\left(\boldsymbol{x}_{2}\right) \\
\vdots \\
\psi_{M-1}\left(\boldsymbol{x}_{N_{\mathrm{ps}}}\right)
\end{array}\right]
$$

$N_{\text {ps }}$ is the number of PS identified in the prior PSI processing (i.e., known locations for the regression fitting). $\mathbf{V}_{m}$ is the data covariance matrix for the PS locations for the $m^{\text {th }}$ interferometric layer. The covariance model is estimated by computing a sample variogram for the residual phase in each interferometric layer $[11,10]$. The best linear unbiased predictor (BLUP) of the atmospheric phase at any 3-D location $\boldsymbol{x}_{0}$ is [11]:

$$
\hat{\psi}_{m}\left(\boldsymbol{x}_{0}\right)=\mathbf{x}_{0}^{T} \hat{\boldsymbol{\beta}}_{m}+\mathbf{v}_{m}^{T} \boldsymbol{V}_{m}^{-1}\left(\boldsymbol{\Psi}_{m}-\mathbf{X} \hat{\boldsymbol{\beta}}_{m}\right)
$$

where $\mathbf{v}_{m}$ is the estimated covariance vector for the atmospheric phases at the location $\boldsymbol{x}_{0}$.

\subsection{Spatio-temporal tomographic inversion}

We use using single-look beamforming (BF) to invert the differential tomographic model $[3,5]$ :

$$
\hat{\alpha}(s, v)=\frac{1}{M} \mathbf{a}^{H}(s, v) \mathbf{y}^{p d} .
$$

where $\alpha$ is the complex scene reflectivity as a function of the unknown elevation-deformation pair $(s, v)$, and $\mathbf{y}^{p d}$ is the observed SAR signal vector for a given range-azimuth pixel, comprising $M$ acquisitions:

$$
\mathbf{y}^{p d}=\left[\begin{array}{llll}
y_{0}^{p d} & y_{1}^{p d} & \ldots & y_{M-1}^{p d}
\end{array}\right]^{T} .
$$

$\mathbf{a}(s, v)$ is the steering vector which is set up such that the atmospheric phase correction is incorporated within the tomographic inversion, as shown below:

$$
\begin{aligned}
& \mathbf{a}(s, v)= \\
& {\left[\begin{array}{c}
\exp \left[-j\left\{\varphi_{0}(s, v)+\hat{\psi}_{0}(\mathbb{T}\{r, a, s\})\right\}\right. \\
\exp \left[-j\left\{\varphi_{1}(s, v)+\hat{\psi}_{1}(\mathbb{T}\{r, a, s\})\right\}\right] \\
\vdots \\
\exp \left[-j\left\{\varphi_{M-1}(s, v)+\hat{\psi}_{M-1}(\mathbb{T}\{r, a, s\})\right\}\right]
\end{array}\right]}
\end{aligned}
$$

where the first phase term in the complex exponential is the interferometric phase model for differential SAR tomography. It is based on the sensor-to-target geometric path-length difference, $\triangle r_{m}(s)$ and assumes the deformation to be a linear function of the temporal baselines, $t_{m}$, as follows:

$$
\varphi_{m}(s, v)=2 k\left[\triangle r_{m}(s)+v t_{m}\right] .
$$

BF-based maximizations under the sequential generalized likelihood ratio test with cancellation (SGLRTC) are applied for the estimation of the unknown scatterer elevation and deformation, as well as for single and double scatterer detections. Details are referred to $[12,5]$.

\section{DATA}

An interferometric stack comprising of 33 Cosmo-SkyMed stripmap images over Matter Valley in the Swiss Alps is used 

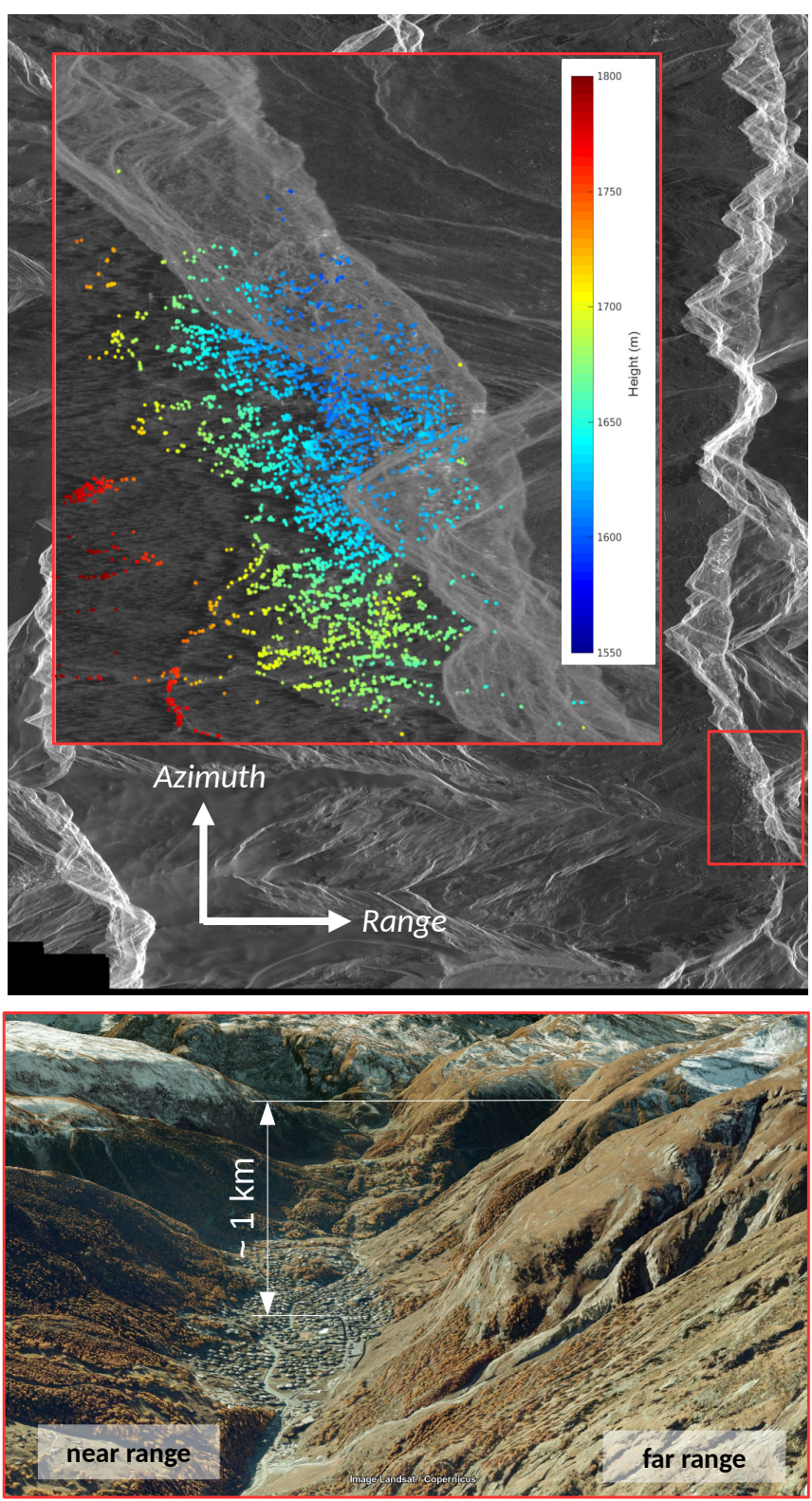

Fig. 1. Top: SAR average intensity for 32 Cosmo-SkyMed acquisitions over Matter Valley in the Swiss Alps. The inset shows the region of interest (ROI) for tomographic analysis. The PS identified in the ROI with the IPTA-bsed PSI processing are shown color-coded. Below: An optical perspective of the ROI, courtesy Google Earth.

in this investigation. The images are taken in the summers of 2008-13 (to avoid temporal decorrelation due to snow cover in winters). Fig. 1 (top) shows an average SAR intensity image of the region. The red colored rectangle encloses the Zermatt village which is our region of interest (ROI) for tomographic analysis. Several settlements in the village are in layover cast by the adjoining mountain. A 3-D perspective of the area is shown in the optical image in Fig. 1 (bottom). We can observe some bare rocks on the mountainside which may exhibit coherent scattering over long term.

\section{RESULTS}

Fig. 1 shows the PS identified in an IPTA-based PSI analysis in the ROI. Fig. 2 shows the single and double scatterers obtained using BF-based spatio-temporal tomographic inversion. Fig. 3 shows the inversion for the specific case of a pixel that contains multiple coherent scatterers.

\section{DISCUSSION \& OUTLOOK}

It can be seen in Fig. 1 that most of the PS are identified in the layover-free area in the village, corresponding to rooftops and other infrastructure. In Fig. 2, we can observe some single scatterers (detected only in the tomographic solution) around $230 \mathrm{~m}$ above the valley floor along the mountainside. A few double scatterers are also detected, but nearly all of them are situated within the built-up area in the village. In this way, spatio-temporal inversion with tomography as an add-on to the PSI solution has increased the deformation sampling. Fig. 3 presents the case of a layover-affected pixel, which is not detected as a PS. When no atmospheric correction is applied, no coherent scatterer is detected. On applying a single correction estimated with spatial filtering of the the atmospheric phases of the neighboring PS (in the valley floor), a single scatterer is detected corresponding to a structure in the valley. When a height-dependent atmospheric correction is applied, we observe multiple scatterers appearing along the elevation profile - the layover is resolved. These results substantiate the applicability and usefulness of the proposed data-driven methodology for the correction of the atmospheric phases for spatio-temporal tomographic inversion in alpine regions.

\section{REFERENCES}

[1] A. Ferretti, C. Prati, and F. Rocca, "Permanent scatterers in SAR interferometry," IEEE Trans. Geosci. Remote Sens., vol. 39, no. 1, pp. 8-20, 2001.

[2] C. Werner, U. Wegmüller, T. Strozzi, and A. Wiesmann, "Interferometric point target analysis for deformation mapping," in Proc. IEEE Int. Geosci. Remote Sens. Symp., 2003, pp. 4362-4364.

[3] F. Lombardini, "Differential tomography: A new framework for SAR interferometry," IEEE Trans. Geosci. Remote Sens., vol. 43, no. 1, pp. $37-44,2005$.

[4] X. Zhu and R. Bamler, "Superresolving SAR tomography for multidimensional imaging of urban areas: Compressive sensing-based TomoSAR inversion," IEEE Signal Process. Mag., vol. 31, no. 4, pp. $51-58,2014$.

[5] M. Siddique, U. Wegmuller, I. Hajnsek, and O. Frey, "Single-Look SAR tomography as an add-on to PSI for improved deformation analysis in urban areas," IEEE Trans. Geosci. Remote Sens., vol. 54, no. 10, pp. 6119-6137, Oct 2016.

[6] S. Tebaldini and A. Guarnieri, "On the role of phase stability in SAR multibaseline applications," IEEE Trans. Geosci. Remote Sens., vol. 48, no. 7, pp. 2953-2966, July 2010. 

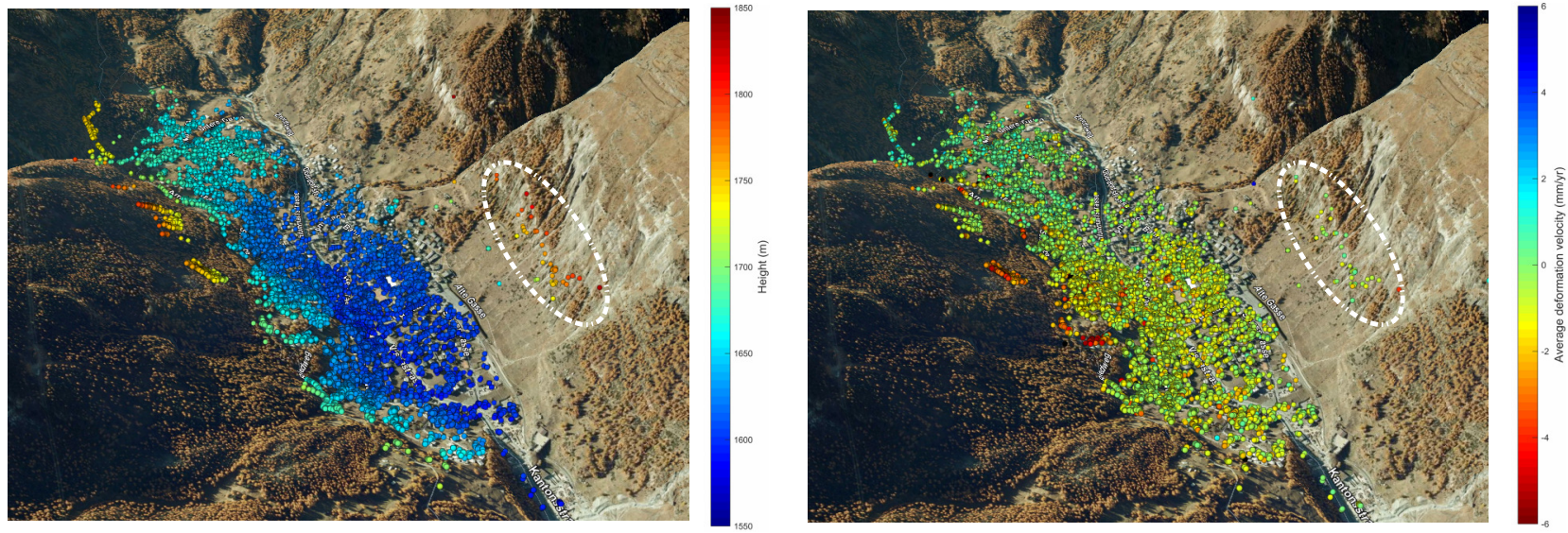

Fig. 2. Geocoded single and double scatterers obtained with tomography. Regression kriging-based height-dependent atmospheric corrections have been applied. Color-coding represents the estimated parameters. Left: Height (m). Right: LOS deformation velocity $(\mathrm{mm} / \mathrm{yr})$.
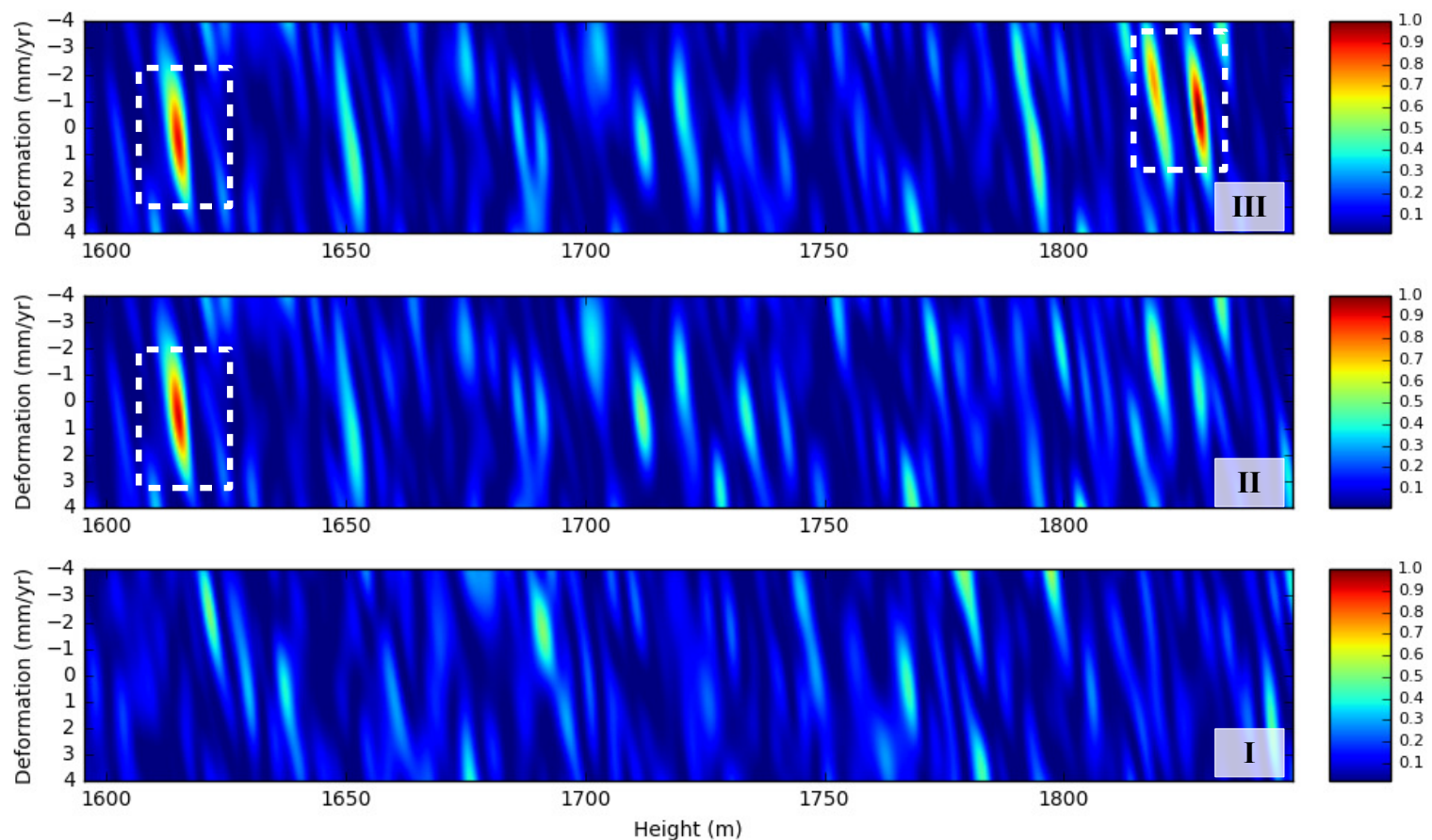

Fig. 3. 2-D squared reflectivity in the height-deformation plane, retrieved with BF-based tomographic inversion, for a layoveraffected pixel. Sub-figure I: No atmospheric correction has been applied. Sub-figure II: A single correction is applied using the spatially filtered atmospheric phase from neighboring PS in the valley. Sub-figure III: Regression kriging-based heightdependent atmospheric corrections are applied. Multiple scatterers are detected in this case, as marked in white.

[7] G. Fornaro, A. Pauciullo, D. Reale, and S. Verde, "Multilook SAR tomography for 3-D reconstruction and monitoring of single structures applied to COSMO-SKYMED data," IEEE J. Sel. Topics Appl. Earth Observ. in Remote Sens., vol. 7, no. 7, pp. 2776-2785, July 2014.

[8] U. Wegmuller, D. Walter, V. Spreckels, and C.L. Werner, "Nonuniform ground motion monitoring with TerraSAR-X persistent scatterer interferometry," IEEE Trans. Geosci. Remote Sens., vol. 48, no. 2, pp. 895-904, Feb 2010.

[9] T. Strozzi, H. Raetzo, U. Wegmuller, J. Papke, R. Caduff, C. Werner, and A. Wiesmann, Satellite and terrestrial radar interferometry for the measurement of slope deformation, pp. 161-165, Springer International Publishing, Cham, 2015.

[10] M. Siddique, I. Hajnsek, and O. Frey, "A case study on the use of differential SAR tomography for measuring deformation in layover areas in rugged alpine terrain," in Proc. IEEE Int. Geosci. Remote Sens. Symp., July 2017, pp. 5850-5853.

[11] R. Bivand, E. Pebesma, and V. Gomez-Rubio, Applied spatial data analysis with $R$, vol. 747248717, Springer, 2008.

[12] A. Pauciullo, D. Reale, A. De Maio, and G. Fornaro, "Detection of double scatterers in SAR tomography," IEEE Trans. Geosci. Remote Sens., vol. 50, no. 9, pp. 3567-3586, 2012. 